\title{
A Compact and UWB Time-delay Line Inspired by CRLH TL Unit Cell
}

\author{
J. Zhang, S.W. Cheung and T.I. Yuk \\ Department of Electrical and Electronic Engineering \\ The University of Hong Kong \\ Hong Kong, China \\ zhangjun@eee.hku.hk, swcheung@eee.hku.hk, tiyuk@eee.hku.hk
}

\begin{abstract}
This paper presents the design of compact and ultrawideband (UWB) time-delay lines inspired by the use of composite right/left-handed transmission line (CRLH TL) unit cell. A conventional CRLH TL unit cell is used as the basic elements for the design of time-delay lines and a rotated version of the unit cell is used to achieve ultrawide bandwidth operation. Time-delay lines for large time delays are designed by cascading the proposed unit cells, optimized using computer simulation and then fabricated on PCBs. A compact UWB time-delay line is designed by bending the time-delay line in a meander. For comparison, the time-delay line using the right-handed transmission line (RH TL) is also fabricated. Simulation and measurement results show that our proposed time-delay lines have ultrawide bandwidth operation and much longer time delays than that of the time-delay line based on RH TL. They also have the advantages of high return losses and low insertion losses.
\end{abstract}

Keywords-compact; UWB; CRLH TL; time-delay lines

\section{INTRODUCTION}

Microwave time-delay lines can find applications in different areas such as radar and phased array systems and other telecommunication systems. For example, time-delay lines are used in the feedforward configuration for analogue predistortion of power amplifiers [1]. In the design of timedelay lines for microwave applications, the size, return loss and insertion loss are all major concerns. Time-delay lines based on waveguides are low loss, but bulky and expensive, while timedelay lines based on transmission lines are of smaller sizes and lower losses.

The concept of metamaterials, commonly known as lefthanded materials (LHMs), was first investigated by Veselago in 1968 [2]. Metamaterials have negative permittivity and permeability which are not commonly found in nature. Although the properties of LHMs promised for a large diversity of novel applications and devices, LHMs did not attract much attention until it was found that the materials could be realized using a general transmission line (TL) approach [3]. Practical LH TLs also have the right-handed (RH) effects, so LHMs realized using TLs are called composite right/left handed transmission lines (CRLH TLs). CRLH TL can be used to design many different microwave components such as phase shifters, antennas and bandpass filters, etc. Recently, timedelay lines based on using the CRLH TL have been proposed and studied [4-7]. However, these designs share one of the major drawbacks, i.e., the bandwidth is relatively narrow.

In this paper, we study the use of the CRLH TL unit cell [4] as the basic elements to design time-delay lines and the rotated version of the unit cell to design time-delay lines for ultra-wide band (UWB) applications. Computer simulation results show that the proposed time-delay line can provide a much longer time delay than the traditional right-handed transmission line (RH TL). A long and narrow time-delay line cannot be easily fitted to practical circuits which normally have limited width and lengths. In this paper, we also propose to bend the wideband time-delay line to form a meander so that the whole time-delay line circuit can be placed on a small rectangular area for easy fitting to practical circuits. Computer simulation using CST MWS 2009 and measurement have been used to study the proposed compact and wideband time-delay lines and results have shown that our proposed time-delay line has an ultra-wide operation bandwidth from $0.1-10 \mathrm{GHz}$, a high return loss of more than $15 \mathrm{~dB}$ and a low insertion loss of less than $1 \mathrm{~dB}$.

\section{TIME-DELAY LINE USING TRANSMISSION LINE}

For a TL with a length of L, the time delay for a signal to travel through is given by:

$$
t_{\text {delay }}=\frac{L}{\lambda f}=\frac{L \times \beta}{2 \pi f}
$$

where $f$ and $\beta$ are the operating frequency and the phase constant, respectively. It can be seen from (1) that the length of the transmission line and the phase constant $\beta$ can be adjusted to produce a longer time delay. For a given time delay, a larger $\beta$ requires a shorter transmission line. Traditional RH TL has a small phase constant $\beta$ and so needs a very long transmission line to produce a large time delay, which leads to a large insertion loss.

\section{MODEL OF CRLH TL UNIT CELL}

The general schematic figure and the equivalent circuit of a CRLH TL unit cell [4] with six fingers are shown in Fig. 1. In Fig. 1(b), the series inductance $L_{R}$ and capacitance $C_{L}$ are the inductance along the fingers and the coupling capacitance 
between the fingers, respectively, the shunt inductance $L_{L}$ is realized by the two fingers each having a via at the ends to the ground, and the shunt capacitance $C_{R}$ is the stray capacitance of the fingers. The expressions for $L_{R}, C_{R}, L_{L}$ and $C_{L}$ are, respectively, given by $[8,9]$ :

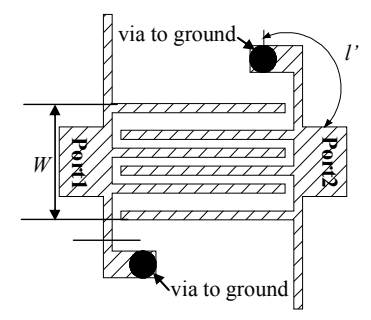

(a)

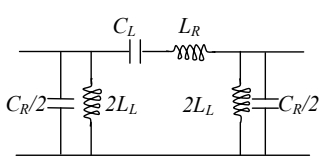

(b)
Figure 1. (a) Structure and (b) equivalent circuit of conventional CRLH TL unit cell

$$
\begin{gathered}
L_{R}=\frac{Z_{0} \sqrt{\varepsilon_{r e}}}{c} l \\
C_{R}=\frac{\sqrt{\varepsilon_{r e}}}{Z_{0} c} l \\
C_{L}=\left(\varepsilon_{r}+1\right) l\left\{4.409(N-3) \tanh \left[0.55\left(\frac{h}{W}\right)^{0.45}\right]\right. \\
\left.\left.\left.+9.92 \tanh \left[0.52\left(\frac{h}{W}\right)^{0.5}\right]\right\} \times 10^{-12}\right]\right) \\
\left.L_{L}=\frac{\mu_{0}\left[h \cdot \ln \left(\frac{h+\sqrt{r^{2}+h^{2}}}{2 \pi}\right)+\frac{3}{2}\left(r-\sqrt{r^{2}+h^{2}}\right)\right]}{r}\right] \\
+\frac{Z_{0} \sqrt{\varepsilon_{r e}}}{c} l^{\prime}
\end{gathered}
$$

where $h$ is the thickness of the substrate, $r$ is the radius of the ground via, $\varepsilon_{r}$ is the relative dielectric constant, $\varepsilon_{r e}$ is the effective dielectric constant, $l$ ' is the distance from the ground via to the port, $l$ is the length of the finger, $N$ is the number of the fingers, $W$ is the total width of all the fingers together and $Z_{0}$ is the characteristic impedance of each of the fingers. The phase constant $\beta$ of such CRLH TL unit cell is [10]:

$$
\beta=\omega \sqrt{L_{R} C_{R}}-\frac{1}{\omega \sqrt{L_{L} C_{L}}}
$$

(2) - (6) show that the structural parameters $L_{R}, C_{R}, L_{L}$ and $C_{L}$ can be used to determine $\beta$ and hence the time delay in (1).

\section{DESIGN OF TIME-DELAY LINES INSPIRED BY CRLH TL UNIT CELL}

\section{A. Narrowband time-delay line}

We propose to use the conventional CRLH TL unit cell as shown in Fig. 1(a) as the basic elements to design time-delay lines. The structural parameters $L_{R}, C_{R}, L_{L}$ and $C_{L}$ through (1)(6) are used to control the value of $\beta$ for the unit cell and hence to maximize the time delay. Then cascading a number of the unit cells together, as shown in Fig.2, can be used to produce a long time delay. However, computer simulation results, as in the following section, show that time-delay lines based the conventional CRLH TL unit cells can provide a large time delay, but have narrow bandwidths.

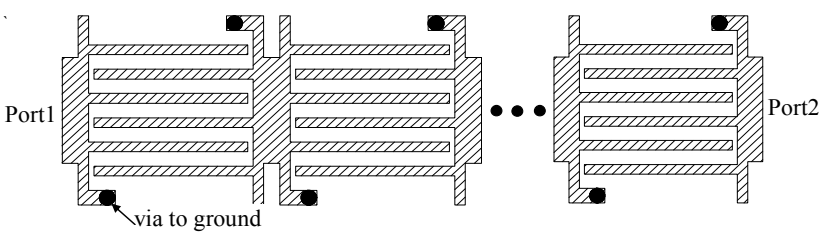

Figure 2. Narrowband time-delay line by cascading conventional CRLH TL unit cells linearly

\section{B. Wideband time-delay line}

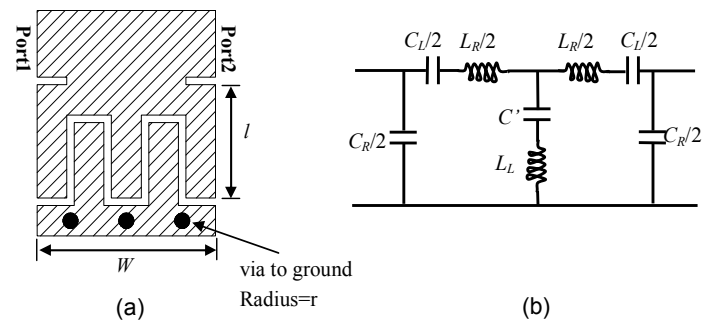

Figure 3. Rotated structure and (b) equivalent circuit of the unit cell

To increase the operation bandwidth of the time-delay line described, we propose to rotate the CRLH TL cell of Fig. 1(a) by 90 -degree, as shown in Fig. 3(a), with the equivalent circuit shown in Fig. 3(b). In this structure, we also propose to reduce the number of fingers to five to make the unit cell having a symmetrical structure. By doing so, the two S-parameters, $\left|S_{11}\right|$ and $\left|S_{21}\right|$ are equal. As a result, we can use identical unit cells in cascade to provide a long time delay and there is no need to perform any matching process between the unit cells.

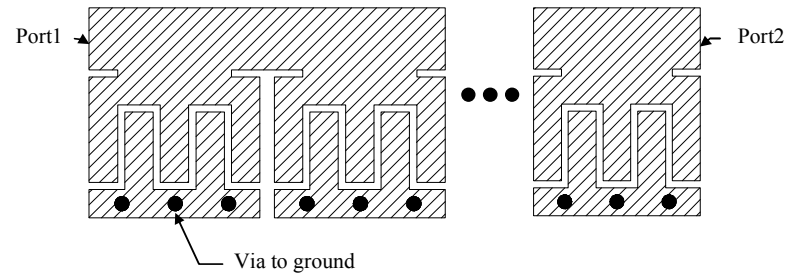

Figure 4. Wideband time-delay line by cascading rotated wideband unit cells linearly

Simulation studies have shown that such unit cell has a much wider bandwidth. A wideband time-delay line constructed using a number of such unit cells cascaded together is shown in Fig. 4. The layouts of the time-delay lines using 16 conventional CRLH TL unit cells and 16 wideband unit cells are shown in Figs. 5(a) and 5(b), respectively.

\section{Compact time-delay line}

As mentioned previously, a long and narrow time-delay line cannot be fitted easily to practical circuits which normally have 
limited width and lengths. Here we propose to bend the wideband time-delay line to form a meander so that the whole time-delay line circuit can be fabricated on a small rectangular area. A compact time-delay line formed by putting 16 wideband unit cells in a meander is shown in Fig. 5(c).

\section{RESULTS AND DISCUSSIONS}

The time-delay lines employing 16 conventional CRLH TL unit cells and 16 wideband unit cells, both having a total length of $60 \mathrm{~mm}$, as shown in Figs. 5(a) and 5(b), respectively, have been designed and optimized using the EM simulation tool CST MWS 2009. In the optimization process, the number of fingers used in each unit cell was fixed at five as shown in Fig. 3 (a) and the width of each finger was set to $0.2 \mathrm{~mm}$ (which is the smallest dimension we could make in our lab). Results have indicated that the length of the fingers in the rotated unit cells has significant effects on $\left|S_{I I}\right|$, so the optimization process mainly optimized the length of the fingers. The dimensions of the unit cell were optimized with the criteria of having $\left|S_{I I}\right|<$ $15 \mathrm{~dB}$ and the largest phase constant $\beta$. The final optimization results were used for fabrication on a F4B-2 PCB with a thickness of $0.8 \mathrm{~mm}$ and permittivity of 2.65 . For comparison, the time-delay line using RH TL with the same length of 60 $\mathrm{mm}$, as shown in Fig. 5(d), has also been fabricated using the same substrate. Finally, the compact wideband time-delay line using 16 wideband unit cells in a meander, as in shown Fig. 5(c), has been designed, optimized, fabricated on a PCB. The photographs of all these prototype time-delay lines are shown in Fig. 6. All these time-delay lines have been measured.

Figure 7 shows the simulated and measured impedance bandwidths (i.e. $\left.\left|S_{I I}\right|\right)$ and insertion losses (i.e., $\left.\left|S_{21}\right|\right)$ of the time-delay lines using the conventional CRLH TL unit cells, the wideband unit cells and the RH TL. The time delays of these time-delay lines are shown in Fig. 8. It can be seen that the simulated and measured results show good agreements. The narrowband time-delay line achieves a time delay of $1800 \mathrm{ps,}$ about $60 \mathrm{ps}(3.5 \%)$ more than that of the wideband time-delay line. However, it has a narrower bandwidth of only 4-6 GHz and a larger insertion loss of about $2 \mathrm{~dB}$ within the 4-6 GHz pass band as shown in Fig. 7(a).

For the wideband time-delay line, Fig. 7(b) shows that the measured return loss is more than $15 \mathrm{~dB}\left(\left|S_{11}\right|<-15 \mathrm{~dB}\right)$ and the insertion loss is less than $1 \mathrm{~dB}$ (i.e., $\left|S_{21}\right|>-1 \mathrm{~dB}$ ) across the whole frequency band from $0.1-10 \mathrm{GHz}$. It achieves a time delay of 1740 ps across the whole frequency band tested, nearly 6 times longer than that of the RH TL, as can be seen in Fig. 8.

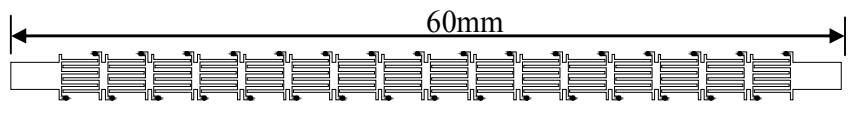

(a)

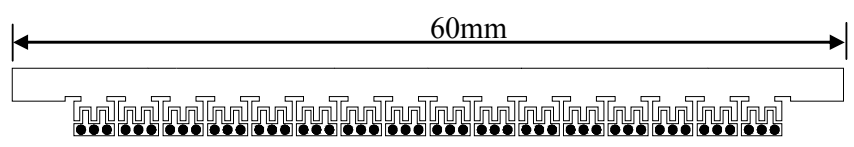

(b)

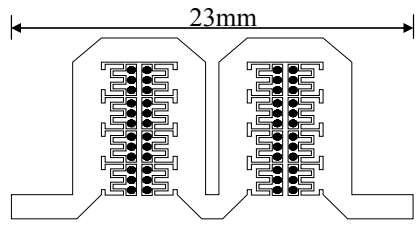

(c)

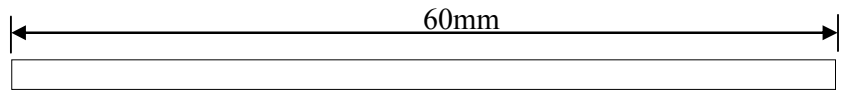

(d)

Figure 5. Layouts of time-delay lines using

(a) conventional CRLH TL unit cells, (b) wideband unit cells, (c) wideband unit cells in a meander and (d) RH TL

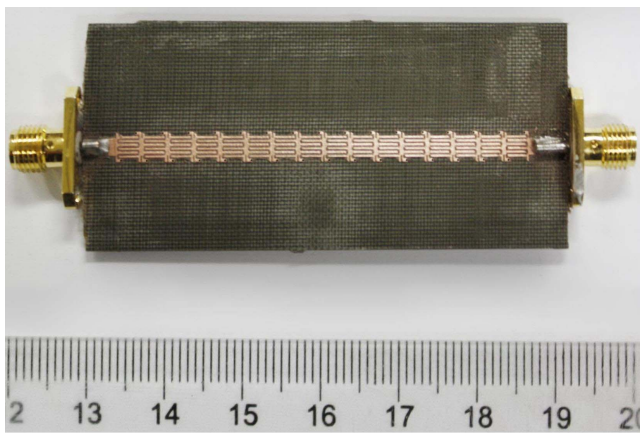

(a)

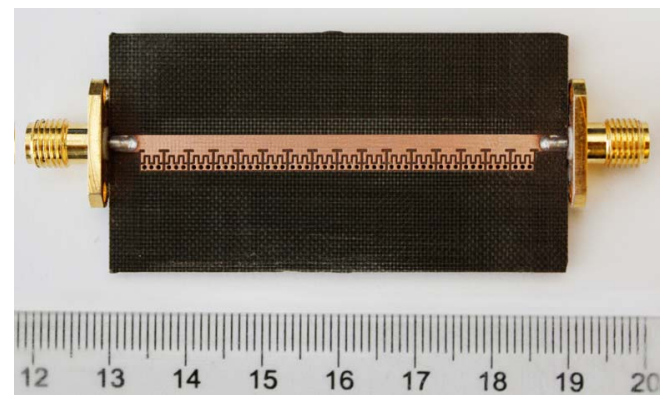

(b)

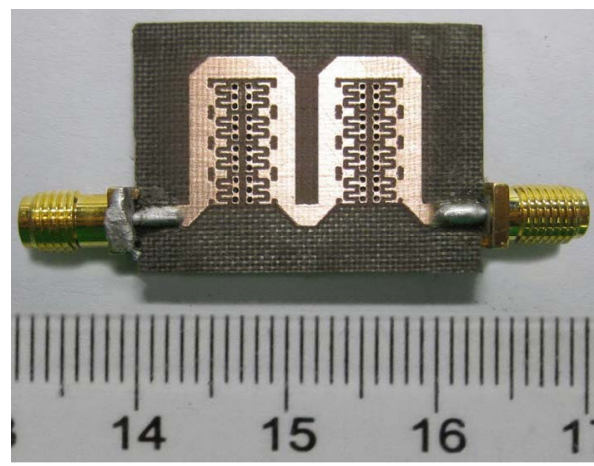

(c) 


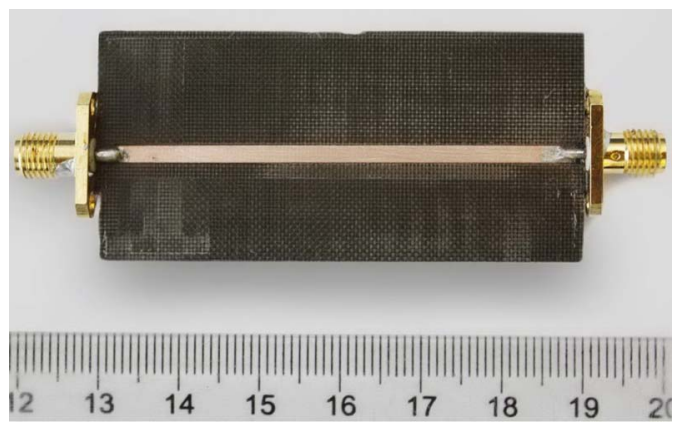

(d)

Figure 6. Photographs of time-delay lines using (a) conventional CRLH TL unit cells, (b) wideband unit cells, (c) wideband unit cells in a meander and (d) RH TL

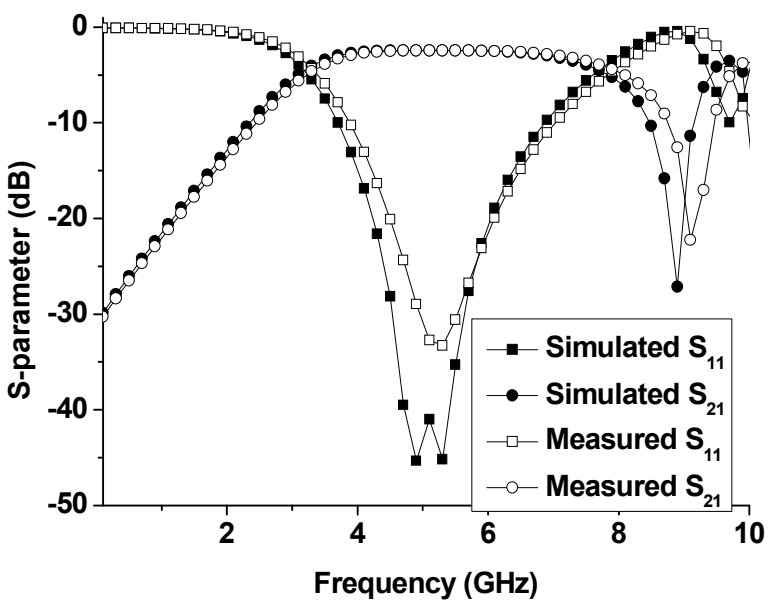

(a)

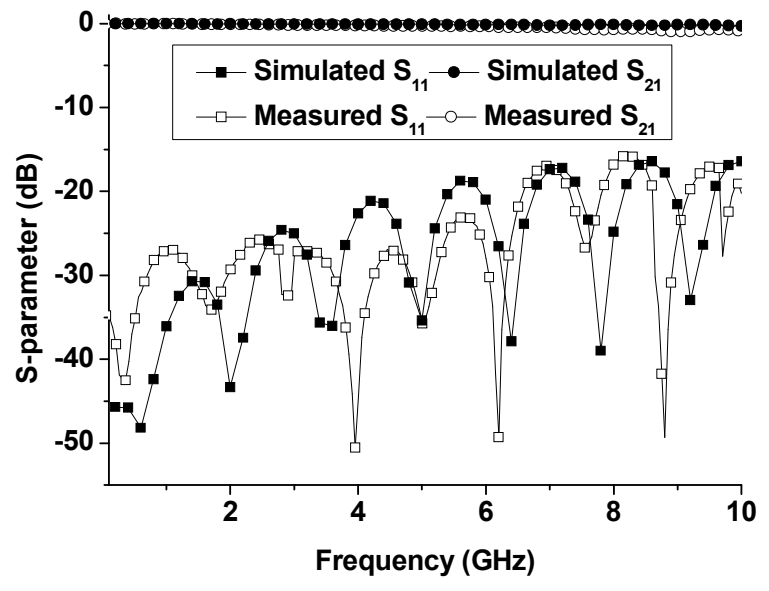

(b)

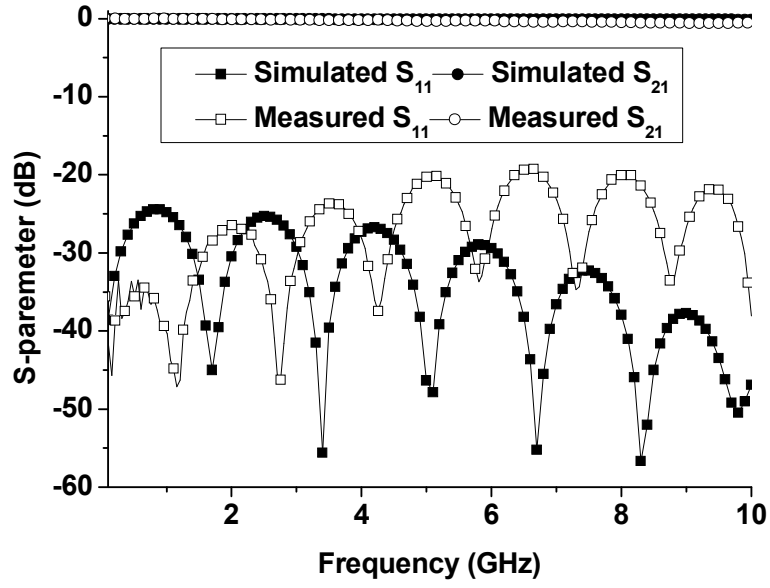

(c)

Figure 7. Simulated and measured $\left|S_{11}\right|$ and $\left|S_{21}\right|$ of time-delay lines using (a) conventional CRLH TL unit cells in linear, (b) wideband unit cells in linear and (c) RH TL

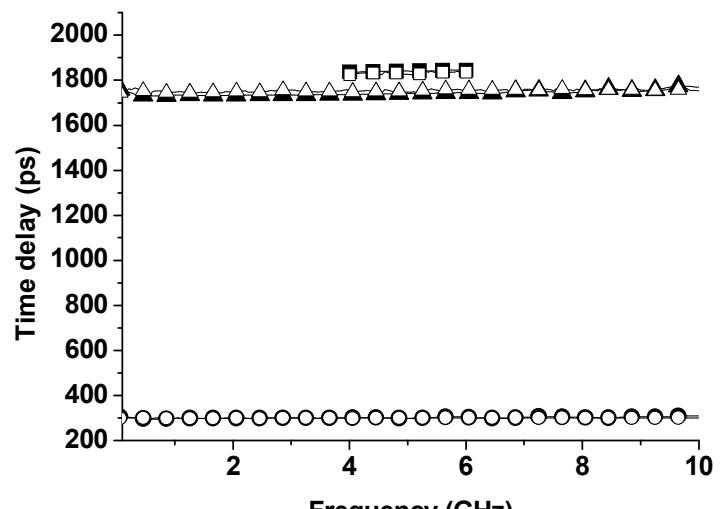

Frequency $(\mathrm{GHz})$

- rotated wideband unit cells in linear(simulated)
- rotated wideband unit cells in linear(measured)
- conventional CRLH TL unit cells in linear(simulated)
- conventional CRLH TL unit cells in linear(measured)
- RH TL(simulated)
- RH TL(measured)

Figure 8. Simulated and measured time delays of different time-delay lines

The time-delay line using the RH TL has quite a high return loss as shown in Fig. 7(c), even higher than that of our proposed wideband time-delay line, and a very low insertion loss of less than $1 \mathrm{~dB}$, but the time delay achieved is only 300 ps which is substantially less than our proposed wideband timedelay line.

For the compact time-delay line of Fig. 6(c), the simulated and measured results on the two S-parameters, $\left|S_{11}\right|$ and $\left|S_{21}\right|$, are shown in Fig. 9 and the time delays are shown in Fig. 10. Again, the simulated and measured results show good agreements. Figure 9 shows that the measured return loss is more than $15 \mathrm{~dB}\left(\left|S_{11}\right|<-15 \mathrm{~dB}\right)$ and insertion loss is less than 1 $\mathrm{dB}$ (i.e., $\left|S_{21}\right|>-1 \mathrm{~dB}$ ) across the whole frequency band tested. 
Thus the meander does not change the properties much. The time delay achieved, as indicated in Fig. 10, is 1820 ps across the whole frequency band of $0.1-10 \mathrm{GHz}$. This is about 6 times more than that of the time-delay line using the RH TL. The time delay is also slightly larger than the wideband timedelay line shown in Fig. 6(b), for the reason that the bending slightly increases the overall length.

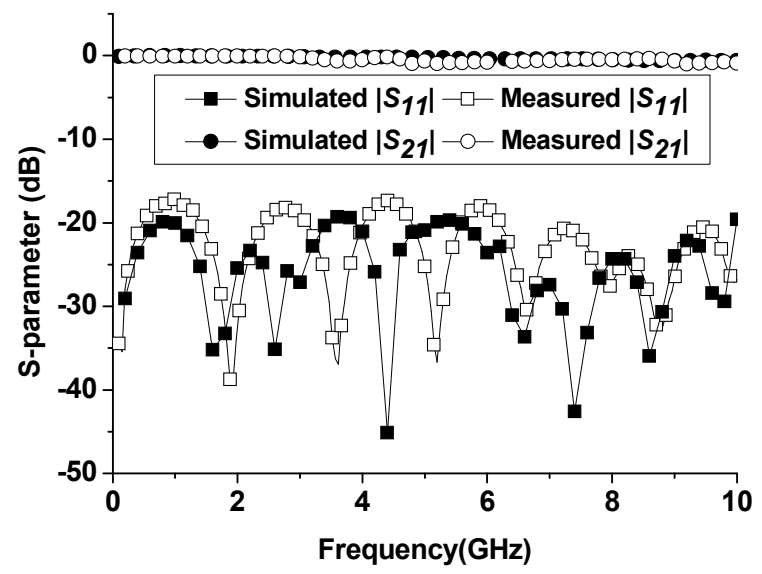

Figure 9. Simulated and measured $\left|S_{I I}\right|$ and $\left|S_{2 I}\right|$ of time-delay line using rotated wideband unit cells in a meander

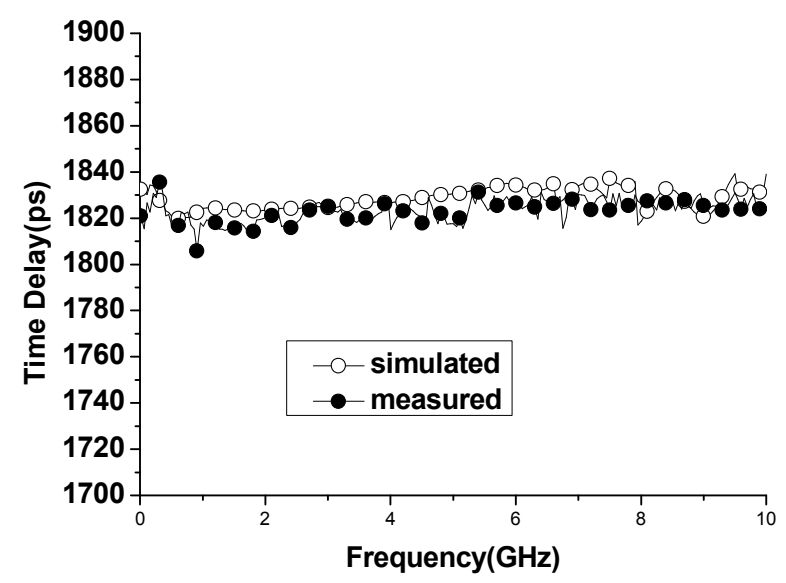

Figure 10. Simulated and measured time delay of time-delay line using rotated wideband unit cells in a meander

\section{CONCLUSIONS}

A compact and UWB time-delay line based on cascading a number of rotated versions of the conventional CRLH TL unit cells in a meander has been proposed, studied and fabricated on a PCB. Three other time-delay lines using the conventional CRLH TL unit cells and RH TL have also been designed, optimized and fabricated for comparison. Simulation and measurement results have demonstrated that, for the length of $60 \mathrm{~mm}$, our proposed compact UWB time-delay line has a much longer time delay of 1820 ps, about 6 times longer than that of using the RH TL, yet having a much wider operating bandwidth of $0.1-10 \mathrm{GHz}$, a high return loss and a low insertion loss.

\section{REFERENCES}

[1] P. B. Kenington: 'High-linearity RF amplifier design' (Norwood, Artech House, 2000), pp. 7-10.

[2] V. Veselago, "The electrodynamics of substances with simultaneously negative values of $\varepsilon$ and $\mu$," Soviet Physics Uspekhi, vol. 10, pp. 509514, 1968.

[3] C. Caloz, H. Okabe, T. Iwai, and T. Itoh, "Transmission line approach of left-handed (LH) materials," Proc. USNC/URSI National Radio Science Meeting, vol. 1, pp. 39, San Antonio, Texas, June 2002,

[4] J. Zhang, Q. Zhu, Q. Jiang and S.J. Xu, "Design of time delay lines with periodic microstrip line and composite right/left-handed transmission line," Microwave and Optical Technology Letters, vol. 51, pp. 16791682, 2009.

[5] S. Pasakawee and Z. Hu, "Left-handed microstrip delay line implemented by Complementary Split Ring Resonators (CSRRs)," in Asia Pacific Microwave Conference 2009, pp. 599-601, Singapore, 2009

[6] Choul-Young Kim, Jaemo Yang, Dong-Wook Kim and Songcheol Hong, "A K-Band CMOS Voltage Controlled Delay Line Based on an Artificial Left-Handed Transmission Line," IEEE Microwave and Wireless Components Letters, vol. 18, pp. 731-733, 2008.

[7] Wenjia Tang and Hongjoon Kim, "Compact, tunable large group delay line," in Wireless and Microwave Technology Conference 2009, Clearwater, FL, 2009.

[8] I. Bahl, Lumped elements for RF and microwave circuits, Artech House: Boston \& London, 2001

[9] E. G. Marc and A. P. Robert, "Modeling via hole grounds in Microstrip," IEEE Microwave and Guided Wave Letters, vol. 1, pp. 135137, 1991.

[10] A. Lai, T. Itoh and C. Caloz, "Composite Right/Left-Handed Transmission Line Metamaterials," IEEE Microwave Magazine, vol. 5, pp. 34-50, 2004. 